\title{
Exercise and obesity in fibromyalgia: beneficial roles of IGF-1 and resistin?
}

Jan L Bjersing ${ }^{1,2^{*}}$, Malin Erlandsson ${ }^{1}$, Maria I Bokarewa ${ }^{1,2}$ and Kaisa Mannerkorpi ${ }^{1,3,4}$

\begin{abstract}
Introduction: Severe fatigue is a major health problem in fibromyalgia (FM). Obesity is common in FM, but the influence of adipokines and growth factors is not clear. The aim was to examine effects of exercise on fatigue, in lean, overweight and obese FM patients.

Methods: In a longitudinal study, 48 FM patients (median 52 years) exercised for 15 weeks. Nine patients were lean (body mass index, BMI 18.5 to 24.9), 26 overweight (BMI 25 to 29.9) and 13 obese. Fatigue was rated on a 0 to $100 \mathrm{~mm}$ scale (fibromyalgia impact questionnaire [FIQ] fatigue) and multidimensional fatigue inventory (MFI-20) general fatigue (MFIGF). Higher levels in FIQ fatigue and MFIGF indicate greater degree of fatigue. Free and total IGF-1, neuropeptides, adipokines were determined in serum and cerebrospinal fluid (CSF).
\end{abstract}

Results: Baseline FIQ fatigue correlated negatively with serum leptin $(r=-0.345 ; P=0.016)$ and nerve growth factor (NGF; $r=-0.412 ; P=0.037)$. In lean patients, baseline MFIGF associated negatively with serum resistin $(r=-0.694$; $P=0.038)$. FIQ Fatigue associated negatively with CSF resistin $(r=-0.365 ; P=0.073)$. Similarly, FIQ fatigue $(r=-0.444$; $P=0.026)$ and MFIGF correlated negatively with CSF adiponectin $(r=-0.508 ; P=0.01)$. In lean patients, FIQ fatigue $(P=0.046)$ decreased after 15 weeks. After 30 weeks, MFIGF decreased significantly in lean (MFIGF: $P=0.017$ ), overweight (MFIGF: $P=0.001$ ), and obese patients (MFIGF: $P=0.016$ ). After 15 weeks, total IGF-1 increased in lean $(P=0.043)$ patients. $\Delta$ Total IGF-1 differed significantly between lean and obese patients $(P=0.010)$. $\Delta$ Total IGF-1 related negatively with $\triangle$ MFIGF after 15 weeks $(r=-0.329 ; P=0.050)$. After 30 weeks, $\triangle F I Q$ fatigue negatively correlated with $\triangle$ NGF $(r=-0.463 ; P=0.034)$ and positively with $\triangle$ neuropeptide $Y(N P Y)(r=0.469 ; P=0.032)$. Resistin increased after 30 weeks $(P=0.034)$. $\triangle$ MFIGF correlated negatively with $\Delta$ resistin $(r=-0.346 ; P=0.031)$, being strongest in obese patients $(r=-0.815 ; P=0.007)$. In obese patients, $\triangle \mathrm{FIQ}$ fatigue after 30 weeks correlated negatively with $\triangle$ free IGF-1 $(r=-0.711 ; P=0.032)$.

Conclusions: Exercise reduced fatigue in all FM patients, this effect was achieved earlier in lean patients. Baseline levels of resistin in both serum and CSF associated negatively with fatigue. Resistin was increased after the exercise period which correlated with decreased fatigue. Changes in IGF-1 indicate similar long-term effects in obese patients. This study shows reduced fatigue after moderate exercise in FM and indicates the involvement of IGF-1 and resistin in these beneficial effects.

Trial registration: ClinicalTrials.gov: NCT00643006

\section{Introduction}

Severe fatigue, together with pain, is a major health problem in fibromyalgia (FM) [1,2] and is considered to be equally important to pain [3] in causing impaired work ability and restricted social participation [4]. It is

\footnotetext{
* Correspondence: jan.bjersing@rheuma.gu.se

'Department of Rheumatology and Inflammation Research, Institute of Medicine, Sahlgrenska Academy, University of Gothenburg, Guldhedsgatan 10, Box 480, 40530 Göteborg, Sweden

Full list of author information is available at the end of the article
}

associated with depression, sleep quality and pain [5]. Obesity is common in FM, with a reported prevalence between 40 and 70\% [6-8]. Increased body mass index (BMI) generally correlates with increased levels of pain and fatigue in FM [7,9-11]. In chronic fatigue syndrome, symptom severity is suggested to be associated with metabolic syndrome [12]. Weight levels may affect neuroendocrine regulation of pain and fatigue through several pathways.

\section{Biomed Central}

(c) 2013 Bjersing et al.; licensee BioMed Central Ltd. This is an open access article distributed under the terms of the Creative Commons Attribution License (http://creativecommons.org/licenses/by/2.0), which permits unrestricted use, distribution, and reproduction in any medium, provided the original work is properly cited. 
There is evidence for deregulation of the growth hormone/insulin-like growth factor (IGF-1) signaling in obesity $[13,14]$ and an inverse relationship between total IGF-1 levels and BMI has been reported $[15,16]$. In FM patients we have recently reported a beneficial role of IGF-1 and exercise with regard to pain [17]. These results were in line with previous findings indicating that IGF-1 has a protective role in FM $[18,19]$ and that IGF-1 promotes resilience to stress and pain in the central nervous system (CNS) [20,21]. Furthermore, growth hormone deficiency is shown to be associated with fatigue and reduced cognitive speed [22].

Recently, several factors, termed adipokines, which are produced in adipose tissue, have been found to have important regulatory roles in both inflammation and nutrition. Adiponectin is one of these adipokines and was initially isolated in adipocytes. Adiponectin regulates energy balance both in peripheral tissues and via the CNS [23,24]. Adiponectin receptors are distributed widely in the brain, affecting appetite, metabolism and autonomic function $[25,26]$. Adiponectin is negatively correlated with depression $[27,28]$ and has antidepressant-like effects in both lean and diet-induced obese mice [29].

Resistin is considered to be an adipokine with unusual properties, and a potential link between inflammation and metabolic disease [30]. It is expressed in human macrophages and has documented regulatory effects on metabolism, adipogenesis and inflammatory reactions [31-33]. Peripheral levels of resistin are upregulated in subjects with insulin resistance and in obesity [34,35], and resistin signaling involves both toll-like receptor (TLR)4 [36] and the IGF-1 receptor [37].

Leptin is another important adipokine. It is a major product of adipose tissue, is increased in obesity and is a central regulator of satiety and body weight $[38,39]$, as well as reproduction, mood and emotion [40-42]. Induction of satiety is mediated by leptin receptors in hypothalamic neuropeptide Y (NPY), producing neurons [43-46]. Leptin has anxiolytic effects in mice $[47,48]$ and is involved in allodynia in a neuropathic pain model [49]. NPY is an abundant neuropeptide, both in the peripheral and in the central nervous system. NPY is an important modulator of hippocampal and thalamic circuits, with the potential to affect a number of different functions in the brain. It is also involved in neuroprotection, neurogenesis and neuroinflammation [50]. NPY is altered in FM patients, possibly involving the hypothalamic-pituitary-adrenal axis [51-54]. NPY is also altered in chronic fatigue syndrome $[55,56]$ and during stress and depression [57]. Disturbed neuropeptide levels with elevated substance P (SP) [58-60] and nerve growth factor (NGF) [61] have previously been found in cerebrospinal fluid in FM. Recent evidence also implicate glial activation in FM with increased IL-6 and IL-8 in cerebrospinal fluid [62].

The aim of the study was to examine the long-term effects of aerobic exercise on fatigue, in lean, overweight, and obese women with FM. Changes in serum free bioactive IGF-1, total IGF-1, IGF binding protein (IGFBP)3, adipokines and neuropeptides were studied to gain a better understanding of the biological mechanisms involved in fatigue in FM.

\section{Materials and methods Study design}

This study is a part of a previously reported randomized controlled exercise study, studying the effects of a moderate-to-high-intensity Nordic walking (NW) program and a supervised low-intensity walking (LIW) program. The effects of Nordic walking on body function were reported previously [63], showing that NW resulted in better improvement in the 6-minute walk test (6MWT) and aerobic capacity, when compared with LIW.

\section{Subjects}

The criteria for inclusion were as follows: women with FM, aged 20 to 60 years, with interest in exercising outdoors twice a week for 15 weeks, who agreed to undergo blood tests at baseline and after the exercise period. To ensure that the patients would manage the planned aerobic exercise, they were required to complete a bicycle test at 50 watts to fulfill the inclusion criteria. All included patients managed to perform the test. They were also invited to participate in an examination of cerebrospinal fluid; however, this was not a criterion for inclusion. FM was defined by the American College of Rheumatology (ACR) 1990 criteria [64]: a history of long-lasting generalized pain and pain in at least 11 of 18 tender points examined by manual palpation.

The criteria for exclusion were as follows: patients who could not speak or read Swedish; presence of other severe somatic or psychiatric disease; BMI <18.5; ongoing or planned physical therapy, including exercise, and inability to attend at the times of the planned exercise sessions.

Forty-nine patients, 26 of them undertaking NW and 23 undertaking LIW, had blood tests at baseline, after 15 weeks of exercise, and at 30 weeks of follow-up, as described in the previous report [17]. One of the patients had BMI $<18.5$ and was therefore excluded from this study. In total, 48 patients with FM formed the study population.

The median age of patients was 52 (48 to 56, interquartile range) years and their median duration of symptoms was 11 ( 7 to 15 ) years. The median number 
of tender points was 15 (13 to 16). Eighty-two percent of patients were taking analgesics during the study and $63 \%$ were taking antidepressants or sedatives. Nine patients were lean (BMI 18.5 to 24.9), 26 patients were overweight (BMI 25.0 to 29.9) and 13 were obese (BMI 230.0). After separating the patients into BMI groups we found a similar distribution in the NW and LIW group. In the lean group, four subjects participated in NW and five in LIW. In the overweight group fifteen participated in NW and eleven in LIW. In the obese group seven participated in NW and six in LIW.

\section{Exercise intervention}

The patients were randomized to either the moderateto-high-intensity NW program $(\mathrm{n}=26)$ or the supervised LIW program $(\mathrm{n}=22)$. Both supervised aerobic exercise programs were conducted twice a week for 40 to 45 minutes for 15 weeks. Patients had blood tests before, after 15 weeks, and after 30 weeks. Pain and fatigue did not significantly change in any of the exercise groups after 15 weeks, while scores in the Multidimensional Fatigue Inventory (MFI-20) [66] subscale of General Fatigue (MFIGF) improved in both groups after 30 weeks. As no differences in fatigue or pain were found between the two exercise groups, and BMI was similarly distributed in both exercise groups, the analyses in this study were conducted on the total population $(n=48)$, irrespective of exercise intensity. Compliance was assessed as attendance at exercise sessions. It was slightly higher among the lean group, whose attendance was $71 \%$, while it was $64 \%$ in the overweight group. Attendance in the obese group was $57 \%$.

\section{Clinical measurements}

Fatigue was rated on a visual analog scale (0 to 100) of the Fibromyalgia Impact Questionnaire (FIQ) [65] which gives an estimation of global fatigue, as well as with the MFIGF [66], which estimates fatigue by questions related to feeling fit, tired and rested. Both instruments reflect fatigue during the last week, and a higher score indicates more severe fatigue.

\section{Blood and cerebrospinal fluid (CSF) sampling}

Serum was collected at rest $(n=48)$ at baseline, after 15 weeks in the exercise program, and at 30 weeks of follow-up $(n=41)$. Serum samples were acquired by venipuncture of the cubital vein. Twenty-six patients agreed to participate in an examination of cerebrospinal fluid (CSF) at baseline. CSF was collected through lumbar puncture through the lumbar vertebrae (L)3/L4 interspace. Collected blood and CSF samples were centrifuged at $800 \mathrm{~g}$ for 3 minutes, aliquoted, and stored frozen at $-70^{\circ} \mathrm{C}$ until use.

\section{Laboratory analyses}

Samples were analyzed with enzyme-linked immunosorbent assay (ELISA) using commercially available kits. Assays specific for human adiponectin (DY1065, $62.5 \mathrm{pg} / \mathrm{ml}$ ), human leptin (DY 398. $31 \mathrm{pg} / \mathrm{ml}$ ), human resistin (DY1359, $31 \mathrm{pg} / \mathrm{ml}$ ), free bioactive IGF-1 (DY291, $4 \mathrm{pg} / \mathrm{ml}$ ) and IGFBP3 (DY675, 0.125ng/ml) were purchased from R and D Systems (Minneapolis, MN, USA). Serum total IGF1 was measured by solidphase, enzyme-labeled chemoluminescent immunoassay (Immulite 2000 IGF1, L2KGF2) on an Immulite 2000 (Siemens Medical Solutions Diagnostics, Los Angeles, CA, USA). An assay specific for NPY (FEK-049-03, $1 \mathrm{pg} / \mathrm{ml}$ ) was purchased from Phoenix Pharmaceuticals (Burlingame, CA, USA). The human NGF-specific assay was purchased from Promega (Madison, WI, USA; $4 \mathrm{pg} / \mathrm{ml}$ ). All assays were run according to recommendations of the manufacturers. Ordinary colorimetric ELISA was read with a Spectramax 340 from Molecular Devices (Sunnyvale, CA, USA), and fluorescent ELISA assays were read with a Mithras LB940 from Berthold Technologies (Bad Wildbad, Germany).

\section{Statistics}

Descriptive data are presented as median and interquartile range. The Wilcoxon signed-rank test was used for comparisons of continuous variables within groups. Baseline data and differences in changes in lean patients were compared by the Mann-Whitney $U$-test with overweight and obese patients, respectively. Relationships between the variables were examined with the Spearman correlation coefficient. To control for possible Type I errors, the upper limit of the number of false significant results was calculated by the following formula:

(Number of tests - Number of significant tests on level of alpha) $*$ Alpha/ (1-Alpha).

\section{Ethics}

The study was approved by the ethics committee of Sahlgrenska University Hospital. Written and verbal information was given to all patients, and written consent was obtained from all patients.

\section{Results}

\section{Relationship between obesity, fatigue, adipokines and}

\section{IGF-1}

Several differences in fatigue and adipokines were found in relation to obesity. Patients with normal BMI (18.5 to 24.9) had higher baseline fatigue $(97 \mathrm{~mm}$ ) compared to overweight patients with BMI 25 to 29.9 (74 mm; $P=0.008$ ), while no significant differences were found compared to obese patients with $\mathrm{BMI} \geq 30(88 \mathrm{~mm}$; $P$-value not significant.) (Table 1 ). Baseline levels of 
Table 1 Clinical data in lean, overweight and obese patients with fibromyalgia

\begin{tabular}{|c|c|c|c|c|c|c|c|c|}
\hline & \multicolumn{2}{|l|}{ Lean (group 1) } & \multicolumn{2}{|c|}{ Overweight (group 2) } & \multicolumn{2}{|c|}{ Obese (group 3) } & \multirow{2}{*}{$\frac{\text { Lean vs overweight }}{P \text {-value }}$} & \multirow{2}{*}{ Lean vs obese } \\
\hline & Median (range) & $\mathrm{n}$ & Median (range) & $\mathrm{n}$ & Median (range) & $n$ & & \\
\hline Age, years & $\begin{array}{c}52.0 \\
(33.5 \text { to } 54.0)\end{array}$ & 9 & $\begin{array}{c}53.0 \\
(48.0 \text { to } 56.0)\end{array}$ & 26 & $51.0(47.0$ to 55.5$)$ & 13 & 0.288 & 0.647 \\
\hline $\mathrm{BMI}, \mathrm{kg} / \mathrm{m}^{2}$ & $\begin{array}{c}23.5 \\
(21.5 \text { to } 24.0)\end{array}$ & 9 & $\begin{array}{c}28.1 \\
\text { (27.1 to } 29.5)\end{array}$ & 26 & 32.7 (30.8 to 35.7 ) & 13 & $<0.001$ & $<0.001$ \\
\hline Tender points, $\mathrm{n}$ & $\begin{array}{c}14.0 \\
(12.5 \text { to } 15.5)\end{array}$ & 9 & $\begin{array}{c}15.0 \\
(13.0 \text { to } 17.0)\end{array}$ & 26 & $15.0(13.0$ to 16.0$)$ & 13 & 0.224 & 0.393 \\
\hline Baseline FIQ fatigue, mm & $\begin{array}{c}97.0 \\
\text { (77.5 to } 98.0)\end{array}$ & 9 & $\begin{array}{c}74.0 \\
\text { (52.8 to } 88.5)\end{array}$ & 26 & 88.0 (72.5 to 91.5$)$ & 13 & 0.008 & 0.110 \\
\hline Baseline MFIGF & $\begin{array}{c}20.0 \\
(17.0 \text { to } 20.0)\end{array}$ & 9 & $\begin{array}{c}16.5 \\
(13.0 \text { to } 20.0)\end{array}$ & 26 & $17.0(14.5$ to 20.0$)$ & 13 & 0.061 & 0.209 \\
\hline $\begin{array}{l}\text { Change in FIQ fatigue after } \\
15 \text { weeks, mm }\end{array}$ & $\begin{array}{c}-7.0 \\
(-13.5 \text { to } 0.0) \\
P=0.046\end{array}$ & 8 & $\begin{array}{c}-0.5 \\
(-16.3 \text { to } 8.3) \\
P=0.485\end{array}$ & 26 & $\begin{array}{c}-8.0(-20.5 \text { to } 2.0) \\
P=0.059\end{array}$ & 13 & 0.368 & 0.972 \\
\hline $\begin{array}{l}\text { Change in FIQ fatigue after } \\
30 \text { weeks, } \mathrm{mm}\end{array}$ & $\begin{array}{c}-4.5 \\
(-12.8 \text { to } 0.3) \\
P=0.161\end{array}$ & 8 & $\begin{array}{c}-2.0(-7.0 \text { to } 8.8) \\
P=0.966\end{array}$ & 24 & $\begin{array}{c}-2.5(-6.8 \text { to } 4.5) \\
\quad P=0.345\end{array}$ & 12 & 0.254 & 0.473 \\
\hline Change in MFIGF after 15 weeks & $\begin{array}{c}-2.0(-4.2 \text { to } 0.0) \\
P=0.084\end{array}$ & 8 & $\begin{array}{c}0.0(-1.2 \text { to } 1.0) \\
P=0.515\end{array}$ & 26 & $\begin{array}{c}0.0(-3.5 \text { to } 1.0) \\
P=0.641\end{array}$ & 13 & 0.164 & 0.336 \\
\hline Change in MFIGF after 30 weeks & $\begin{array}{c}-3.0 \\
(-5.5 \text { to }-2.0) \\
P=0.017\end{array}$ & 8 & $\begin{array}{c}-2.0(-3.0 \text { to } 0.0) \\
P=0.001\end{array}$ & 24 & $\begin{array}{l}-3.0(-4.0 \text { to }-1.0) \\
P=0.016\end{array}$ & 12 & 0.147 & 0.624 \\
\hline
\end{tabular}

Levels of fatigue (FIQ and MFIGF) at baseline ( 0 weeks), during training (15 weeks) and after training (30 weeks). Lean patients had BMI 18.5 to 24.9 ; overweight patients had BMI 25.0 to 29.9. Obese patients had BMI $\geq 30.0$. Median values and interquartile range are indicated. ${ }^{\mathrm{a}} \mathrm{Mann}$-Whitney $U$-test. $\mathrm{n}$, number; BMI, body mass index; FIQ, Fibromyalgia Impact Questionnaire; MFIGF, Multidimensional Fatigue Inventory subscale of General Fatigue.

adiponectin were higher in lean compared to overweight patients $(P=0.013)$ and compared to obese patients $(P=0.003)$ (Table 2$)$. Leptin levels were lowest in lean patients and tended to be higher in overweight patients $(P=0.067)$ and were highest in obese patients $(P<0.001)$. Resistin levels did not differ significantly between groups. Total IGF-1 was higher in lean patients compared to overweight patients (160.0 vs $113.0 \mathrm{ng} / \mathrm{ml}$, $P=0.026)$ and obese patients $(160.0$ vs 106.5 , $P=0.056$ ), (Table 3 ). Serum free IGF-1 and IGFB3 did not differ between the groups.

Fatigue was also related to adipokine levels and IGF-1 levels. Baseline fatigue was negatively correlated with serum levels of leptin $(r=-0.345, P=0.016, \mathrm{n}=48)$ and NGF $(r=-0.412, P=0.037, \mathrm{n}=26)$ (Table 4). Leptin correlated negatively with total IGF-1 $(r=-0.354$, $P=0.020, \mathrm{n}=43)$ and positively with NPY $(r=0.472$, $P=0.015, \mathrm{n}=26)$ and NGF. Serum free IGF-1 correlated with total IGF-1 $(\mathrm{r}=0.366 ; P=0.016 ; \mathrm{n}=43)$ and IGFB3 ( $\mathrm{r}=0.361 ; P=0.016 ; \mathrm{n}=44)$ and with NGF $(\mathrm{r}=0.401 ; P=0.042 ; \mathrm{n}=26)$. In lean patients, baseline fatigue was negatively associated with resistin levels ( $\mathrm{r}=-0.694 ; P=0.038 ; \mathrm{n}=9$ ).

FIQ Fatigue was negatively associated with resistin levels in CSF ( $r=-0.365, P=0.073, \mathrm{n}=25)$ (Table 5). A similar pattern was seen for CSF levels of adiponectin with negative correlations to FIQ fatigue $(r=-0.444, P=0.026$, $\mathrm{n}=25)$ and MFIGF $(r=-0.508, P=0.01, \mathrm{n}=25)$.

\section{Influence of exercise on fatigue}

In the group as a whole, FIQ fatigue was decreased after 15 weeks (median -4 , interquartile range -15 to 4 , $P=0.024, \mathrm{n}=47)$, and after 30 weeks both FIQ fatigue $(-2,-7$ to $4.5 ; P=0.252, \mathrm{n}=44)$ and MFIGF were decreased $(-2,-4$ to $-1, P<0.001, \mathrm{n}=44)$. In lean patients, FIQ fatigue $(-7,13.5$ to $0, P=0.046)$ and MFIGF (-2, -4.2 to $0, P=0.084)$ were decreased after 15 weeks (Table 1). After 30 weeks, MFIGF decreased significantly in lean patients, $(-3,-5.5$ to $-2, P=0.017)$, overweight patients $(-2,-3$ to $0, P=0.001)$ and obese patients $(-3,-4$ to $-1, P=0.016)$, and the direction of change in FIQ fatigue was the same although not significant.

\section{Influence of exercise on levels of IGF-1, adipokines and neuropeptides}

As mentioned above, total IGF-1 was highest in lean patients and lower in overweight and obese patients (Table 3). After 15 weeks, total IGF-1 was further increased in lean patients $(33 \mathrm{ng} / \mathrm{ml}, 0$ to $51, P=0.043, \mathrm{n}$ $=7)$, but was unchanged in overweight patients $(10,-13.5$ to $19, P=0.309, \mathrm{n}=17)$ and in obese patients $(-1,-22$ to $4.5, P=0.255)$. The change in total IGF-1 differed significantly between lean and obese patients $(P=0.010)$. After 30 weeks, serum free IGF-1 was significantly decreased in obese patients $(-2.1 \mathrm{ng} / \mathrm{ml},-4.7$ to $-0.1, P=0.017, \mathrm{n}=10)$ and in overweight patients $(-0.7,-1.6$ to $0.6, P=0.053$, 
Table 2 Adipokines in lean, overweight and obese patients

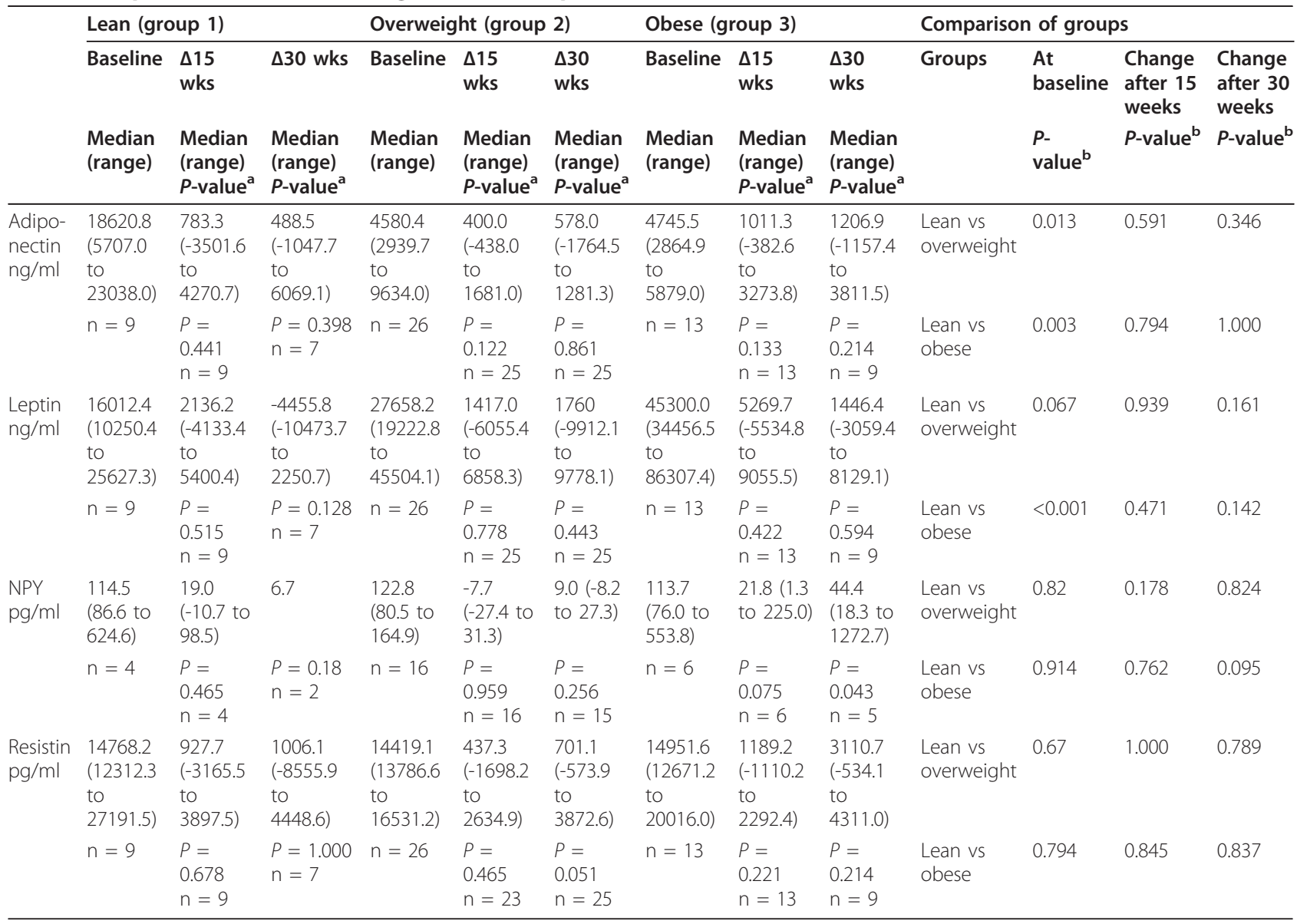

Serum levels of adiponectin, leptin, NPY and resistin at baseline ( 0 weeks), change $(\Delta)$ during training (15 weeks) and change after training ( 30 weeks). Median values and interquartile range are indicated. ${ }^{a}$ Wilcoxon signed rank test. ${ }^{b}$ Mann-Whitney $U$-test. NPY, neuropeptide Y.

$\mathrm{n}=24$ ) but was unchanged in lean FM patients. Resistin increased in the group as a whole after 30 weeks $(944 \mathrm{pg} /$ $\mathrm{ml},-819$ to $4299, P=0.034, \mathrm{n}=41)$, while adiponectin and leptin were unchanged after 30 weeks. NPY levels were increased after 30 weeks $(11.1,0.6$ to $33, P=0.017$, $\mathrm{n}=22)$, this increase was only significant in obese patients $(44.4,18.3$ to $1272, P=0.043, \mathrm{n}=5)$. Adiponectin levels were increased in the whole group of FM patients after 15 weeks $(695,-432$ to $1891, P=0.022$, $\mathrm{n}=47$ ) but not after 30 weeks.

\section{Changes in fatigue in relation to IGF-1, adipokines and neuropeptides}

Change in MFIGF ( $\triangle$ MFIGF) after 15 weeks was negatively correlated with $\Delta$ total IGF-1 $(r=-0.329, P=0.050, \mathrm{n}=36)$. In lean patients, $\Delta$ total IGF-1 was correlated with $\Delta$ resistin in serum $(r=0.829, P=0.021, \mathrm{n}=7)$ after 15 weeks; $\Delta$ free IGF-1 after 15 weeks of exercise correlated positively with $\triangle$ NGF in serum $(r=0.428, P=0.029, \mathrm{n}=26)$.

After 30 weeks, $\Delta$ free IGF-1 was negatively correlated with $\triangle$ NPY $(r=-0.563, P=0.006)$ (Table 6). $\Delta$ FIQ fatigue was correlated negatively with $\triangle \mathrm{NGF}(r=-0.463$, $P=0.034, \mathrm{n}=21)$ and positively with $\triangle \mathrm{NPY}(r=0.469, P$ $=0.032, \mathrm{n}=21) . \Delta$ MFIGF correlated negatively with $\Delta$ resistin $(r=-0.346, P=0.031, \mathrm{n}=39)$; this negative correlation was strong in obese patients $(r=-0.815$, $P=0.007, \mathrm{n}=9$ ) (Table 7) but was much weaker in lean and overweight patients. In obese patients, $\triangle$ FIQ fatigue after 30 weeks was negatively correlated with $\Delta$ free IGF-1 $(r=-0.711, P=0.032, \mathrm{n}=9)$ and $\Delta$ adiponectin $(\mathrm{r}=-0.753, P=0.019)($ Table 7$)$.

\section{Type 1 error}

Analyses of baseline data, changes in fatigue, levels of adipokines and IGF levels (Tables 1, 2, 3, and text) comprised a total of 121 comparisons and the upper level of the number of false significant results was 5.10, which means that five of the significant results might be false.

Correlations at baseline (Tables 4 and 5, and text), comprised a total of 57 comparisons and the upper level of the number of false significant results was 2.26 , which means that two significant results might be false. 
Table 3 Serum free IGF-1, total IGF-1 and IGFB3 in lean, overweight and obese patients

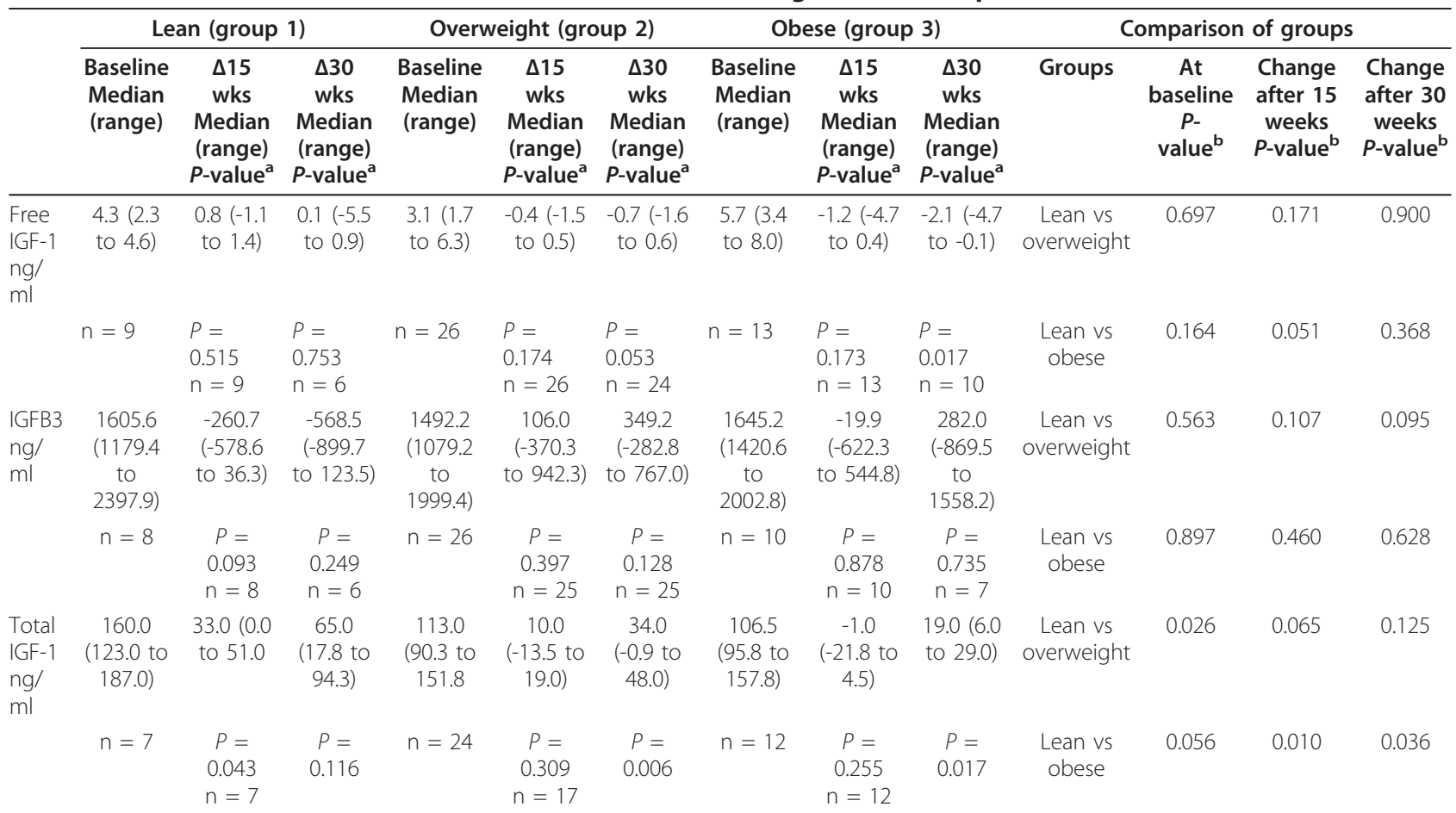

Levels of serum free IGF-1, total serum IGF-1 and serum IGFB3 at baseline (0 weeks), change ( $\Delta$ ) during training (15 weeks) and change after training ( 30 weeks). Median values and interquartile range are indicated. ${ }^{a}$ Wilcoxon signed rank test. ${ }^{b}$ Mann-Whitney $U$-test. IGF-1, insulin-like growth factor-1; IGFB3, insulin-like growth factor-binding protein-3.

Table 4 Fatigue versus adipokines, IGF-1 and neuropeptides

\begin{tabular}{|c|c|c|c|c|c|c|c|c|c|c|}
\hline & & FIQ fatigue & Adiponectin & Leptin & Resistin & Free IGF-1 & Total IGF-1 & IGFB3 & NPY & NGF \\
\hline MFIGF & $\begin{array}{l}r \\
p \\
\mathrm{n}\end{array}$ & $\begin{array}{c}0.623 \\
<0.001 \\
48\end{array}$ & $\begin{array}{c}0.107 \\
0.468 \\
48\end{array}$ & $\begin{array}{c}-0.074 \\
0.618 \\
48\end{array}$ & $\begin{array}{c}-0.125 \\
0.398 \\
48\end{array}$ & $\begin{array}{c}0.024 \\
0.871 \\
48\end{array}$ & $\begin{array}{c}0.254 \\
0.100 \\
43\end{array}$ & $\begin{array}{c}0.039 \\
0.801 \\
44\end{array}$ & $\begin{array}{c}0.150 \\
0.464 \\
26\end{array}$ & $\begin{array}{c}0.141 \\
0.493 \\
26\end{array}$ \\
\hline FIQ Fatigue & $\begin{array}{l}r \\
p \\
\mathrm{n}\end{array}$ & $\begin{array}{c}1.000 \\
48\end{array}$ & $\begin{array}{c}0.143 \\
0.333 \\
48\end{array}$ & $\begin{array}{c}-0.345 \\
0.016 \\
48\end{array}$ & $\begin{array}{c}-0.111 \\
0.451 \\
48\end{array}$ & $\begin{array}{c}-0.032 \\
0.829 \\
48\end{array}$ & $\begin{array}{c}0.198 \\
0.203 \\
43\end{array}$ & $\begin{array}{c}0.155 \\
0.315 \\
44\end{array}$ & $\begin{array}{c}0.004 \\
0.984 \\
26\end{array}$ & $\begin{array}{c}-0.412 \\
0.037 \\
26\end{array}$ \\
\hline Adiponectin & $\begin{array}{l}r \\
p \\
\mathrm{n}\end{array}$ & & $\begin{array}{c}1.000 \\
48\end{array}$ & $\begin{array}{c}0.032 \\
0.827 \\
48\end{array}$ & $\begin{array}{c}0.123 \\
0.405 \\
48\end{array}$ & $\begin{array}{c}-0.200 \\
0.172 \\
48\end{array}$ & $\begin{array}{c}0.148 \\
0.343 \\
43\end{array}$ & $\begin{array}{c}-0.143 \\
0.355 \\
44\end{array}$ & $\begin{array}{c}0.245 \\
0.227 \\
26\end{array}$ & $\begin{array}{c}-0.151 \\
0.460 \\
26\end{array}$ \\
\hline Leptin & $\begin{array}{l}r \\
p \\
\mathrm{n}\end{array}$ & & & $\begin{array}{c}1.000 \\
48\end{array}$ & $\begin{array}{c}0.153 \\
0.300 \\
48\end{array}$ & $\begin{array}{c}0.131 \\
0.374 \\
48\end{array}$ & $\begin{array}{c}-0.354 \\
0.020 \\
43\end{array}$ & $\begin{array}{c}0.082 \\
0.595 \\
44\end{array}$ & $\begin{array}{c}0.472 \\
0.015 \\
26\end{array}$ & $\begin{array}{c}0.426 \\
0.030 \\
26\end{array}$ \\
\hline Resistin & $\begin{array}{l}r \\
P \\
\mathrm{n}\end{array}$ & & & & $\begin{array}{c}1.000 \\
48\end{array}$ & $\begin{array}{c}-0.103 \\
0.486 \\
48\end{array}$ & $\begin{array}{c}0.036 \\
0.817 \\
43\end{array}$ & $\begin{array}{c}-0.043 \\
0.782 \\
44\end{array}$ & $\begin{array}{c}0.023 \\
0.912 \\
26\end{array}$ & $\begin{array}{c}0.148 \\
0.470 \\
26\end{array}$ \\
\hline Free IGF-1 & $\begin{array}{l}r \\
p \\
\mathrm{n}\end{array}$ & & & & & $\begin{array}{c}1.000 \\
48\end{array}$ & $\begin{array}{c}0.366 \\
0.016 \\
43\end{array}$ & $\begin{array}{c}0.361 \\
0.016 \\
44\end{array}$ & $\begin{array}{c}0.167 \\
0.414 \\
26\end{array}$ & $\begin{array}{c}0.401 \\
0.042 \\
26\end{array}$ \\
\hline Total IGF-1 & $\begin{array}{l}r \\
p \\
\mathrm{n}\end{array}$ & & & & & & $\begin{array}{c}1.000 \\
43\end{array}$ & $\begin{array}{c}0.188 \\
0.250 \\
39\end{array}$ & $\begin{array}{c}0.085 \\
0.706 \\
22\end{array}$ & $\begin{array}{c}0.233 \\
0.296 \\
22\end{array}$ \\
\hline IGFB3 & $\begin{array}{l}r \\
p \\
\mathrm{n}\end{array}$ & & & & & & & $\begin{array}{c}1.000 \\
44\end{array}$ & $\begin{array}{c}0.395 \\
0.046 \\
26\end{array}$ & $\begin{array}{c}0.198 \\
0.332 \\
26\end{array}$ \\
\hline $\begin{array}{l}\text { Neuropeptide Y } \\
\text { (NPY) }\end{array}$ & $\begin{array}{l}r \\
P \\
\mathrm{n}\end{array}$ & & & & & & & & $\begin{array}{l}1.000 \\
26\end{array}$ & $\begin{array}{c}0.340 \\
0.089 \\
26\end{array}$ \\
\hline
\end{tabular}

Correlation between baseline fatigue (FIQ and MFIGF), serum free IGF-1, serum levels of total IGF-1, IGFB3, adipokines and neuropeptides. $r$, Spearman's correlation coefficient; $P, P$-value; n, number; MFIGF, Multidimensional Fatigue Inventory subscale of General Fatigue; FIQ, Fibromyalgia Impact Questionnaire; IGF, insulin-like growth factor; IGFB3, insulin-like growth factor-binding protein-3; NGF, nerve growth factor. 
Table 5 Correlation between baseline fatigue and cerebrospinal fluid levels of adipokines and neuropeptides

\begin{tabular}{|c|c|c|c|c|c|c|}
\hline & MFIGF & Adiponectin CSF & Leptin CSF & Resistin CSF & NPY CSF & NGF CSF \\
\hline FIQ fatigue & $\begin{array}{c}r=0.623 \\
P<0.001 \\
\mathrm{n}=48\end{array}$ & $\begin{array}{c}r=-0.444 \\
P=0.026 \\
\mathrm{n}=25\end{array}$ & $\begin{array}{c}r=-0.233 \\
P=0.263 \\
\mathrm{n}=25\end{array}$ & $\begin{array}{c}r=-0.365 \\
P=0.073 \\
\mathrm{n}=25\end{array}$ & $\begin{array}{c}r=-0.111 \\
P=0.607 \\
\mathrm{n}=24\end{array}$ & $\begin{array}{c}r=-0.243 \\
P=0.243 \\
\mathrm{n}=25\end{array}$ \\
\hline MFIGF & & $\begin{array}{c}r=-0.508 \\
P=0.01 \\
n=25\end{array}$ & $\begin{array}{c}r=-0.189 \\
P=0.365 \\
n=25\end{array}$ & $\begin{array}{c}r=-0.316 \\
P=0.123 \\
n=25\end{array}$ & $\begin{array}{c}r=0.014 \\
P=0.947 \\
\mathrm{n}=24\end{array}$ & $\begin{array}{c}r=0.219 \\
P=0.293 \\
n=25\end{array}$ \\
\hline
\end{tabular}

Correlation between baseline fatigue (FIQ and MFIGF) and cerebrospinal fluid levels of adiponectin, leptin, resistin, NPY and NGF. $r$, Spearman's correlation coefficient; $P$, $P$-value; $\mathrm{n}$, number; FIQ, Fibromyalgia Impact Questionnaire; MFIGF, Multidimensional Fatigue Inventory subscale of General Fatigue; CSF, cerebrospinal fluid; NPY, neuropeptide Y; NGF, nerve growth factor.

Table 6 Change in fatigue versus change in adipokines, IGF-1 and neuropeptides

\begin{tabular}{|c|c|c|c|c|c|c|c|c|c|c|}
\hline & & $\begin{array}{l}\Delta \text { MFIGF } \\
30 \text { wks }\end{array}$ & $\begin{array}{c}\Delta \text { Adiponectin } \\
30 \text { wks }\end{array}$ & $\begin{array}{l}\Delta \text { Leptin } \\
30 \text { wks }\end{array}$ & $\begin{array}{c}\Delta \text { Resistin } \\
30 \text { wks }\end{array}$ & $\begin{array}{c}\Delta \text { Free IGF-1 } \\
30 \text { wks }\end{array}$ & $\begin{array}{c}\Delta \text { Total IGF-1 } \\
30 \text { wks }\end{array}$ & $\begin{array}{l}\Delta \text { IGFB3 } \\
30 \text { wks }\end{array}$ & $\begin{array}{l}\Delta \text { NGF } \\
30 \text { wks }\end{array}$ & $\begin{array}{c}\Delta \mathrm{NPY} \\
30 \text { wks }\end{array}$ \\
\hline $\begin{array}{l}\triangle \mathrm{FIQ} \text { fatigue } \\
30 \text { wks }\end{array}$ & $\begin{array}{l}r \\
p \\
\mathrm{n}\end{array}$ & $\begin{array}{c}0.415 \\
0.005 \\
44\end{array}$ & $\begin{array}{c}-0.227 \\
0.164 \\
39\end{array}$ & $\begin{array}{c}-0.111 \\
0.502 \\
39\end{array}$ & $\begin{array}{c}-0.016 \\
0.923 \\
39\end{array}$ & $\begin{array}{c}-0.147 \\
0.385 \\
37\end{array}$ & $\begin{array}{c}0.106 \\
0.549 \\
34\end{array}$ & $\begin{array}{c}0.250 \\
0.141 \\
36\end{array}$ & $\begin{array}{c}-0.463 \\
0.034 \\
21\end{array}$ & $\begin{array}{c}0.469 \\
0.032 \\
21\end{array}$ \\
\hline $\begin{array}{l}\triangle M F I G F \\
30 w k s\end{array}$ & $\begin{array}{l}r \\
p \\
\mathrm{n}\end{array}$ & $\begin{array}{c}1.000 \\
44\end{array}$ & $\begin{array}{c}-0.096 \\
0.561 \\
39\end{array}$ & $\begin{array}{c}0.280 \\
0.084 \\
39\end{array}$ & $\begin{array}{c}-0.346 \\
0.031 \\
39\end{array}$ & $\begin{array}{c}-0.201 \\
0.232 \\
37\end{array}$ & $\begin{array}{c}-0.075 \\
0.672 \\
34\end{array}$ & $\begin{array}{c}0.382 \\
0.022 \\
36\end{array}$ & $\begin{array}{c}0.043 \\
0.852 \\
21\end{array}$ & $\begin{array}{c}0.209 \\
0.364 \\
21\end{array}$ \\
\hline $\begin{array}{l}\triangle \text { Adiponectin } \\
30 \text { wks }\end{array}$ & $\begin{array}{l}r \\
p \\
\mathrm{n}\end{array}$ & & $\begin{array}{c}1.000 \\
41\end{array}$ & $\begin{array}{c}-0.016 \\
0.922 \\
41\end{array}$ & $\begin{array}{c}0.011 \\
0.943 \\
41\end{array}$ & $\begin{array}{c}0.002 \\
0.989 \\
39\end{array}$ & $\begin{array}{c}0.091 \\
0.600 \\
36\end{array}$ & $\begin{array}{c}-0.241 \\
0.145 \\
38\end{array}$ & $\begin{array}{c}0.097 \\
0.669 \\
22\end{array}$ & $\begin{array}{c}-0.361 \\
0.099 \\
22\end{array}$ \\
\hline $\begin{array}{l}\Delta \text { Leptin } \\
30 \text { wks }\end{array}$ & $\begin{array}{l}r \\
p \\
\mathrm{n}\end{array}$ & & & $\begin{array}{c}1.000 \\
41\end{array}$ & $\begin{array}{c}-0.009 \\
0.954 \\
41\end{array}$ & $\begin{array}{c}-0.211 \\
0.196 \\
39\end{array}$ & $\begin{array}{c}0.051 \\
0.765 \\
36\end{array}$ & $\begin{array}{c}-0.058 \\
0.730 \\
38\end{array}$ & $\begin{array}{c}0.199 \\
0.374 \\
22\end{array}$ & $\begin{array}{c}-0.091 \\
0.687 \\
22\end{array}$ \\
\hline $\begin{array}{l}\Delta \text { Resistin } \\
30 \text { wks }\end{array}$ & $\begin{array}{l}r \\
p \\
\mathrm{n}\end{array}$ & & & & $\begin{array}{c}1.000 \\
41\end{array}$ & $\begin{array}{c}0.048 \\
0.770 \\
39\end{array}$ & $\begin{array}{c}-0.186 \\
0.277 \\
36\end{array}$ & $\begin{array}{c}-0.143 \\
0.393 \\
38\end{array}$ & $\begin{array}{c}0.103 \\
0.647 \\
22\end{array}$ & $\begin{array}{c}-0.278 \\
0.210 \\
22\end{array}$ \\
\hline $\begin{array}{l}\Delta \text { Free IGF-1 } \\
30 \text { wks }\end{array}$ & $\begin{array}{l}r \\
p \\
\mathrm{n}\end{array}$ & & & & & $\begin{array}{c}1.000 \\
40\end{array}$ & $\begin{array}{c}0.103 \\
0.556 \\
35\end{array}$ & $\begin{array}{c}0.178 \\
0.299 \\
36\end{array}$ & $\begin{array}{c}0.356 \\
0.104 \\
22\end{array}$ & $\begin{array}{c}-0.563 \\
0.006 \\
22\end{array}$ \\
\hline $\begin{array}{l}\Delta \text { Total IGF-1 } \\
30 \text { wks }\end{array}$ & $\begin{array}{l}r \\
p \\
n\end{array}$ & & & & & & $\begin{array}{c}1.000 \\
40\end{array}$ & $\begin{array}{c}0.043 \\
0.812 \\
33\end{array}$ & $\begin{array}{c}-0.115 \\
0.639 \\
19\end{array}$ & $\begin{array}{c}-0.128 \\
0.601 \\
19\end{array}$ \\
\hline $\begin{array}{l}\triangle 1 G F B 3 \\
30 w k s\end{array}$ & $\begin{array}{l}r \\
p \\
\mathrm{n}\end{array}$ & & & & & & & $\begin{array}{l}1.000 \\
38\end{array}$ & $\begin{array}{c}-0.089 \\
0.695 \\
22\end{array}$ & $\begin{array}{c}-0.242 \\
0.277 \\
22\end{array}$ \\
\hline $\begin{array}{l}\triangle \mathrm{NGF} \\
30 \mathrm{wks}\end{array}$ & $\begin{array}{l}r \\
p \\
n\end{array}$ & & & & & & & & $\begin{array}{l}1.000 \\
22\end{array}$ & $\begin{array}{c}-0.215 \\
0.336 \\
22\end{array}$ \\
\hline
\end{tabular}

Correlation after 30 weeks between change $(\Delta)$ in fatigue ( $\triangle F I Q$ and $\triangle$ MFIGF) and change in serum free IGF-1, serum levels of IGFB3, adipokines and neuropeptides. $r$, Spearman's correlation coefficient; $P, P$-value; $\mathrm{n}$, number; FIQ, Fibromyalgia Impact Questionnaire; MFIGF, Multidimensional Fatigue Inventory subscale of General Fatigue; IGF, insulin-like growth factor; IGFB3, insulin-like growth factor-binding protein-3; NGF, nerve growth factor; NPY, neuropeptide Y.

Correlations with regard to change (Tables 6 and 7 , and text), comprised a total of 93 comparisons and the upper level of number of false significant results was 4.21 , which means that four significant results might be false.

\section{Discussion}

Fatigue is a debilitating and common health problem in FM and in many autoimmune rheumatic diseases, influencing quality of life, work ability and motivation to exercise. The cause of fatigue is multifactorial and poorly understood. Suggested causes of chronic fatigue include central and peripheral neuropeptides and cytokines, endocrine dysregulation and secondary effects due to pain, depression and sleep disturbance $[3,18,55,56,67]$.

Aerobic exercise, together with pharmacological treatment, is one of the cornerstones of treatment for FM [68], and many patients with FM report lower levels of fatigue after a lengthy exercise period [63,69]. In this group of women with FM, the response to aerobic exercise on fatigue was related to levels of BMI. Lean patients already reported significantly reduced fatigue after 15 weeks of exercise. The response to exercise in overweight and obese patients was delayed, but a significant reduction in fatigue was found after six months. An association 
Table 7 Change in fatigue in lean, overweight and obese patients versus change in adipokines, IGF-1 and neuropeptides

\begin{tabular}{|c|c|c|c|c|c|c|c|c|c|c|}
\hline \multicolumn{2}{|l|}{ Lean patients } & $\begin{array}{c}\begin{array}{c}\Delta \text { MFIGF } \\
30 \text { wks }\end{array} \\
0.268\end{array}$ & $\begin{array}{c}\begin{array}{c}\Delta \text { Adiponectin } \\
\text { 30 wks }\end{array} \\
0.541\end{array}$ & $\begin{array}{c}\Delta \text { Leptin } \\
30 \text { wks }\end{array}$ & $\begin{array}{c}\Delta \text { Resistin } \\
\mathbf{3 0} \text { wks } \\
-0.685\end{array}$ & $\begin{array}{c}\Delta \text { Free IGF-1 } \\
\text { 30 wks } \\
0.145\end{array}$ & $\begin{array}{c}\begin{array}{c}\Delta \text { Tot IGF-1 } \\
30 \text { wks }\end{array} \\
0.812\end{array}$ & $\begin{array}{c}\Delta \text { IGFB3 } \\
30 \text { wks }\end{array}$ & $\begin{array}{c}\begin{array}{c}\Delta \mathrm{NPY} \\
\mathbf{3 0} \mathrm{wks}\end{array} \\
1.000\end{array}$ & \multirow{2}{*}{$\begin{array}{c}\Delta \text { NGF } \\
30 \text { wks } \\
\begin{array}{c}1.000 \\
2\end{array}\end{array}$} \\
\hline $\begin{array}{l}\triangle \mathrm{FIQ} \text { Fatigue } \\
30 \text { weeks }\end{array}$ & $\begin{array}{l}r \\
p \\
\mathrm{n}\end{array}$ & $\begin{array}{c}0.268 \\
0.521 \\
8\end{array}$ & $\begin{array}{c}0.541 \\
0.210 \\
7\end{array}$ & $\begin{array}{c}-0.432 \\
0.333 \\
7\end{array}$ & $\begin{array}{c}-0.685 \\
0.090 \\
7\end{array}$ & $\begin{array}{c}0.145 \\
0.784 \\
6\end{array}$ & $\begin{array}{c}0.812 \\
0.05 \\
6\end{array}$ & $\begin{array}{c}0.086 \\
0.872 \\
6\end{array}$ & $\begin{array}{c}1.000 \\
2\end{array}$ & \\
\hline $\begin{array}{l}\triangle M F I G F \\
30 \text { weeks }\end{array}$ & $\begin{array}{l}r \\
p \\
\mathrm{n}\end{array}$ & $\begin{array}{c}1.000 \\
8\end{array}$ & $\begin{array}{l}0.275 \\
0.550 \\
7\end{array}$ & $\begin{array}{c}-0.220 \\
0.635 \\
7\end{array}$ & $\begin{array}{c}-0.239 \\
0.606 \\
7\end{array}$ & $\begin{array}{c}0.059 \\
0.912 \\
6\end{array}$ & $\begin{array}{c}0.706 \\
0.117 \\
6\end{array}$ & $\begin{array}{c}0.177 \\
0.738 \\
6\end{array}$ & $\begin{array}{c}1.000 \\
2\end{array}$ & $\begin{array}{c}1.000 \\
2\end{array}$ \\
\hline Overweight $p$ & nts & $\begin{array}{l}\Delta \text { MFIGF } \\
30 \text { wks }\end{array}$ & $\begin{array}{c}\Delta \text { Adiponectin } \\
30 \text { wks }\end{array}$ & $\begin{array}{l}\Delta \text { Leptin } \\
30 \text { wks }\end{array}$ & $\begin{array}{c}\Delta \text { Resistin } \\
30 \text { wks }\end{array}$ & $\begin{array}{c}\Delta \text { Free IGF-1 } \\
30 \text { wks }\end{array}$ & $\begin{array}{c}\Delta \text { Tot IGF-1 } \\
30 \text { wks }\end{array}$ & $\begin{array}{l}\text { IGFB3 } \\
30 \text { wks }\end{array}$ & $\begin{array}{l}\Delta \text { NPY } \\
30 \text { wks }\end{array}$ & $\begin{array}{l}\Delta \text { NGF } \\
30 \text { wks }\end{array}$ \\
\hline $\begin{array}{l}\triangle F I Q \text { Fatigue } \\
30 \text { wks }\end{array}$ & $\begin{array}{l}r \\
p \\
\mathrm{n}\end{array}$ & $\begin{array}{c}0.530 \\
0.008 \\
24\end{array}$ & $\begin{array}{c}-0.219 \\
0.315 \\
23\end{array}$ & $\begin{array}{c}-0.099 \\
0.654 \\
23\end{array}$ & $\begin{array}{c}0.270 \\
0.213 \\
23\end{array}$ & $\begin{array}{c}-0.021 \\
0.924 \\
22\end{array}$ & $\begin{array}{c}0.213 \\
0.380 \\
19\end{array}$ & $\begin{array}{c}0.260 \\
0.232 \\
23\end{array}$ & $\begin{array}{c}0.394 \\
0.164 \\
14\end{array}$ & $\begin{array}{c}-0.475 \\
0.086 \\
14\end{array}$ \\
\hline $\begin{array}{l}\triangle M F I G F \\
30 w k s\end{array}$ & $\begin{array}{l}r \\
p \\
\mathrm{n}\end{array}$ & $\begin{array}{l}1.000 \\
24\end{array}$ & $\begin{array}{c}-0.089 \\
0.685 \\
23\end{array}$ & $\begin{array}{c}0.127 \\
0.562 \\
23\end{array}$ & $\begin{array}{c}-0.265 \\
0.222 \\
23\end{array}$ & $\begin{array}{c}-0.233 \\
0.297 \\
22\end{array}$ & $\begin{array}{c}-0.012 \\
0.962 \\
19\end{array}$ & $\begin{array}{c}0.104 \\
0.635 \\
23\end{array}$ & $\begin{array}{c}0.384 \\
0.176 \\
14\end{array}$ & $\begin{array}{c}-0.047 \\
0.874 \\
14\end{array}$ \\
\hline Obese patient & & $\begin{array}{l}\Delta \text { MFIGF } \\
30 \text { wks }\end{array}$ & $\begin{array}{c}\Delta \text { Adiponectin } \\
30 \text { wks }\end{array}$ & $\begin{array}{l}\Delta \text { Leptin } \\
30 \text { wks }\end{array}$ & $\begin{array}{c}\Delta \text { Resistin } \\
30 \text { wks }\end{array}$ & $\begin{array}{c}\Delta \text { Free IGF-1 } \\
30 \text { wks }\end{array}$ & $\begin{array}{c}\Delta \text { Tot IGF-1 } \\
30 \text { wks }\end{array}$ & $\begin{array}{l}\Delta \text { IGFB3 } \\
30 \text { wks }\end{array}$ & $\begin{array}{l}\Delta \text { NPY } \\
30 \text { wks }\end{array}$ & $\begin{array}{l}\Delta \text { NGF } \\
30 \text { wks }\end{array}$ \\
\hline $\begin{array}{l}\Delta \text { FIQ Fatigue } \\
30 \text { wks }\end{array}$ & $\begin{array}{l}r \\
p \\
n\end{array}$ & $\begin{array}{c}0.283 \\
0.373 \\
12\end{array}$ & $\begin{array}{c}-0.753 \\
0.019 \\
9\end{array}$ & $\begin{array}{c}0.084 \\
0.831 \\
9\end{array}$ & $\begin{array}{c}-0.326 \\
0.391 \\
9\end{array}$ & $\begin{array}{c}-0.711 \\
\mathbf{0 . 0 3 2} \\
9\end{array}$ & $\begin{array}{c}-0.261 \\
0.498 \\
9\end{array}$ & $\begin{array}{c}0.072 \\
0.878 \\
7\end{array}$ & $\begin{array}{c}0.154 \\
0.805 \\
5\end{array}$ & $\begin{array}{c}-0.205 \\
0.741 \\
5\end{array}$ \\
\hline $\begin{array}{l}\triangle M F I G F \\
30 w k s\end{array}$ & $\begin{array}{l}r \\
p \\
\mathrm{n}\end{array}$ & $\begin{array}{c}1.000 \\
12\end{array}$ & $\begin{array}{c}-0.210 \\
0.587 \\
9\end{array}$ & $\begin{array}{c}0.647 \\
0.060 \\
9\end{array}$ & $\begin{array}{c}-0.815 \\
0.007 \\
9\end{array}$ & $\begin{array}{c}-0.025 \\
0.949 \\
9\end{array}$ & $\begin{array}{c}-0.418 \\
0.263 \\
9\end{array}$ & $\begin{array}{c}0.786 \\
0.036 \\
7\end{array}$ & $\begin{array}{c}-0.900 \\
\mathbf{0 . 0 3 7} \\
5\end{array}$ & $\begin{array}{c}0.500 \\
0.391 \\
5\end{array}$ \\
\hline
\end{tabular}

Correlation after 30 weeks between change $(\Delta)$ in fatigue ( $\triangle$ FIQ and $\Delta$ MFIGF) and change in serum free IGF-1, serum levels of IGFB3, adipokines and neuropeptides in lean patients, overweight and obese patients. $r$, Spearman's correlation coefficient; $P, P$-value; $\mathrm{n}$, number; FIQ, Fibromyalgia Impact Questionnaire; MFIGF, Multidimensional Fatigue Inventory subscale of General Fatigue; IGF, insulin-like growth factor; IGFB3, insulin-like growth factor-binding protein-3; NGF, nerve growth factor; NPY, neuropeptide Y.

between BMI and fatigue in FM has previously been reported [70], and a high BMI together with inactivity also increases the risk for development of FM [71]. In our material, the overweight group reported lower levels of fatigue than the lean group. Fatigue levels between the lean and obese groups did not significantly differ.

We used two different instruments to rate fatigue [72]. Both ratings of general fatigue reflect symptom severity, but somewhat different aspects. The FIQ rates the global feeling of fatigue, possibly including a feeling of pain, and the MFIGF estimates fatigue in relation to feeling fit, tired and rested.

We found evidence of a positive role for total and free bioactive IGF-1 on fatigue. This is in line with previous reports that IGF-1 has a protective role in FM $[17-19,73]$ promoting adaptation and neuroplasticity in the central nervous system [20,21]. Baseline levels of resistin in CSF were negatively correlated with fatigue. The same pattern, although not significant, was seen for resistin in serum. Increased resistin after 6 months correlated with reduced fatigue. Thus, the increase in resistin during exercise appears to improve fatigue, and the positive effects may be especially important in obese patients. Resistin represents a potential link between inflammation and metabolism and can stimulate TLR4 [36] as well as promote IGF-1 receptor signaling [37].
To the best of our knowledge, resistin has not previously been studied in relation to fatigue.

We also found evidence of a role for adiponectin, leptin and NPY in the reduced fatigue after exercise. Serum leptin and cerebrospinal adiponectin were both associated with low fatigue at baseline, and change in adiponectin correlated with reduced fatigue. NPY correlated with increased fatigue. Serum leptin is taken up via the blood-brain barrier and is a central regulator of energy levels with behavioral effects $[43,42,48]$. The arcuate nucleus of the hypothalamus is believed to be important in mediating these effects [43,74]. Different peripheral energy signals such as leptin and insulin [74] were found to activate different but overlapping subpopulations of arcuate NPY neurons. In line with this, the IGF-1-receptor is expressed in arcuate neurons and glial cells [75], and IGF-1 receptor activation is important for neuroplasticity in the arcuate hypothalamus [76]. Similarly, resistin can activate hypothalamic neurons and induce NPY expression in the hypothalamus [77]. Based on our findings, the roles of leptin and NPY in fatigue and the long-term effects of exercise merit further study.

This is an exploratory longitudinal study. Since we aimed to investigate the interaction of IGF-1 and adipokines in relation to BMI, the study includes many analyses. Due to multiple analyses, the significance level 
should be interpreted with caution, and the upper limit of the expected number of false sigificant results is presented in the Results section.

\section{Conclusions}

Aerobic exercise reduced fatigue in all FM patients; this effect was achieved early in lean patients. In overweight and obese patients the reduction of fatigue was most pronounced after 6 months. Fatigue in FM patients is inversely correlated to resistin in serum and CSF, indicating a beneficial role of resistin. The long-term reduction of fatigue following exercise correlated with increased levels of resistin. The inverse correlation of resistin with reduced fatigue was more pronounced in obese FM patients. Changes in IGF-1 indicate a similar beneficial role on fatigue in obese patients. The results also indicate the involvement of leptin, adiponectin and NPY, although it is not clear how these signals may interact with each other in chronic fatigue.

\begin{abstract}
Abbreviations
ACR: American College of Rheumatology; BMI: body mass index; CNS: central nervous system; CSF: cerebrospinal fluid; ELISA: enzyme-linked immunosorbent assay; FM: fibromyalgia; FIQ: Fibromyalgia Impact Questionnaire; IL: interleukin; IGF-1: insulin-like growth factor-1; IGFBP3: insulin-like growth factor-binding protein-3; L3/L4: lumbar vertebrae 3 to 4; LIW: low-intensity walking; MFI-20: Multidimensional Fatigue Inventory; MFIGF: Multidimensional Fatigue Inventory subscale of General Fatigue; 6MWT: 6-minute walking test; NGF: nerve growth factor; NPY: neuropeptide Y; NW: Nordic walking; SP: substance P; TLR: toll-like receptor.
\end{abstract}

\section{Authors' contributions \\ JB: study conception and design, analysis and interpretation of data. ME: analysis and interpretation of data. MBo: acquisition, analysis and interpretation of data. KM: study conception, study design, acquisition, analysis and interpretation of data. All the authors were involved in the drafting of the article and revising it critically for important intellectual content. All the authors approved the final version of the article.}

\section{Competing interests}

The authors declare that they have no competing interests.

\section{Acknowledgements}

We thank Lena Nordeman, Åsa Cider, Gunilla Jonsson and all other members of the GAU-study group for recruiting, examining or supervising the exercise groups. This work has been funded by grants from the Swedish Research Council (KM, MBo), the Medical Society of Göteborg (MBo), the Swedish Association against Rheumatism (KM, MBo), the King Gustaf V:s 80year Foundation (MBo), Professor Nanna Swartz Foundation (MBo), Torsten Söderberg's Foundation (MBo), Rune and Ulla Amlövs Trust, the Swedish Research Agency for Innovation Systems (VINNOVA), the Swedish Foundation for Strategic Research, the Ingabritt and Arne Lundberg's Foundation, Magnus Bergwall Foundation (MBo), the University of Göteborg, the Family Thölen and Kristlers Foundation, the Regional agreement on medical training and clinical research between the Western Götaland county council and the University of Göteborg (LUA/ALF) (JB, KM, MBo), the Health and Medical Care Executive Board of Västra Götaland Region (KM). The funding sources have no involvement in study design; in the collection, analysis and interpretation of data; in the writing of the report; and in the decision to submit the article for publication.

\section{Author details}

'Department of Rheumatology and Inflammation Research, Institute of Medicine, Sahlgrenska Academy, University of Gothenburg, Guldhedsgatan
10, Box 480, 40530 Göteborg, Sweden. 'Sahlgrenska University Hospital, Rheumatology. Gröna stråket 14. 41345 Göteborg. Sweden. ${ }^{3}$ Sahlgrenska University Hospital, Physiotherapy and Occupational therapy. Vita stråket 13 . 41345 Göteborg. Sweden. ${ }^{4}$ University of Gothenburg Centre for Personcentered Care (GPCC), Sahlgrenska Academy, Gothenburg, Sweden.

Received: 21 November 2012 Revised: 29 January 2013

Accepted: 21 February 2013 Published: 27 February 2013

\section{References}

1. Aaron LA, Burke MM, Buchwald D: Overlapping conditions among patients with chronic fatigue syndrome, fibromyalgia, and temporomandibular disorder. Arch Intern Med 2000, 160:221-227.

2. Mease P, Clauw D, Christensen R, Crofford L, Gendreau R, Martin SA, Simon LS, Strand V, Williams DA, Arnold LM, OMERACT Fibromyalgia Working Group: Toward development of a fibromyalgia responder index and disease activity score: OMERACT module update. J Rheumatol 2011, 38:1487-1495.

3. Staud R: Peripheral and central mechanisms of fatigue in inflammatory and noninflammatory rheumatic diseases. Curr Rheumatol Rep 2012, 14:539-548.

4. Mannerkorpi K, Gard G: Hinders for continued work among persons with fibromyalgia. BMC Muskuloskeletal Disorders 2012, 13:96.

5. Nicassio P, Moxham E, Schumand C, Gevirtz R: The contribution of pain, reported sleep quality, and depressive symptoms to fatigue in fibromyalgia. Pain 2002, 100:271-279.

6. Bennet RM, Jones J, Turk DC, Russel IJ, Matallana L: An internet survey of 2,596 people with fibromyalgia. BMC Musculoskelet Disorders 2007, 8:27.

7. Neumann L, Lerner E, Glazer Y, Bolotin A, Shefer A, Buskila D: A crosssectional study of the relationship between body mass index and clinical characteristics, tenderness measures, quality of life and physical functioning in fibromyalgia patients. Clin Rheumatol 2008, 27:1543-1547.

8. Okifuji A, Bradshaw DH, Olson C: Evaluating obesity in fibromyalgia: neuroendocrine biomarkers, symptoms and functions. Clin Rheumatol 2009, 28:475-478.

9. Kim CH, Luedtke CA, Vincent A, Thompson JM, Oh TH: Association of body mass index with symptom severity and quality of life in patients with fibromyalgia. Arthritis Care Res 2012, 64:222-228.

10. Mork PJ, Vasseljen O, Nilsen Tl: Association between physical exercise, body mass index and risk of fibromyalgi: longitudinal data from the Norwegian Nord-Trondelag Health Study. Arthritis Care Res 2010, 62:611-617.

11. Okifuji A, Donaldson GW, Barck L, Fine PG: Relationship between fibromyalgia and obesity in pain function, mood and sleep. J Pain 2010, 11:1329-1337.

12. Maloney EM, Boneva RS, Lin JM, Reeves WC: Chronic fatigue syndrome is associated with metabolic syndrome: results from a case-control study in Georgia. Metabolism 2010, 59:1351-1357.

13. Vahl N, Jørgensen JO, Skjaerbaek C, Veldhuis JD, Orskov H, Christiansen JS: Abdominal adiposity rather than age and sex predicts mass and regularity of GH secretion in healthy adults. Am J Physiol 1997, 272: E1108-E1116.

14. Pijl H, Langendonk JG, Burggraaf J, Frölich M, Cohen AF, Veldhuis JD, Meinders AE: Altered neuroregulation of $\mathrm{GH}$ secretion in viscerally obese premenopausal women. J Clin Endocrinol Metab 2001, 86:5509-5515.

15. Faupel-Badger JM, Berrigan D, Ballard-Barbash R, Potischman N: Anthropometric corelates of insulin-like growth factor 1 (IGF-1) and IGF1 binding protein-3 (IGFBP-3) levels by race/ethnicity and gender. Ann Epidemiol 2009, 19:841-849.

16. Friedrich $N$, Jørgensen $T$, Juul A, Spielhagen C, NaucK M, Wallaschofski $H$, Linneberg A: Insulin-like growth factor I and anthropometric parameters in a Danish population. Exp Clin Endocrinol Diabetes 2012, 120:171-174.

17. Bjersing $J$, Dehlin M, Erlandsson M, Bokarewa Ml, Mannerkorpi K: Changes in pain and insulin-like growth factor 1 in fibromyalgia during exercise: the involvement of cerebrospinal inflammatory factors and neuropeptides. Arthr Res Ther 2012, 14:R162.

18. Jones KD, Deodhar P, Lorentzen A, Bennett RM, Deodhar AA: Growth hormone perturbations in fibromyalgia: a review. Semin Arthritis Rheum 2007, 36:357-379.

19. Cuatrecasas G, Alegre C, Fernandez-Sola J, Gonzalez MJ, Garcia-Fructuoso F, Poca-Dias V, Nadal A, Navarro F, Mera A, Lage M, Peino R, Casanueva F, 
Linan C, Sesmilo G, Coves MJ, Izquierdo JP, Alvarez I, Granados E, PuigDomingo M: Growth hormone treatment for sustained pain reduction and improvement in quality of life in severe fibromyalgia. Pain 2012, 153:1382-1389.

20. Garcia-Segura LM, Diz-Chaves Y, Perez-Martin M, Darnaudéry M: Estradiol, insulin-like growth factor-I and brain aging. Psychoneuroendocrinology 2007, 32:S57-S61.

21. Morgado C, Silva L, Pereira-Terra P, Tavares I: Changes in serotonergic and noradrenergic descending pain pathways during painful diabetic neuropathy: the preventive action of IGF1. Neurobiol Dis 2011, 43:275-284.

22. Arwert LI, Veltman DJ, Deijen JB, van Dam PS, Delemarre-van deWaal HA, Drent ML: Growth hormone deficiency and memory functioning in adults visualized by functional magnetic resonance imaging. Neuroendocrinology 2005, 82:32-40.

23. Thundyil J, Pavloski D, Sobey CG, Arumugam TV: Adiponectin receptor signalling in the brain. Br J Pharmacol 2012, 165:313-327.

24. Yamauchi T, Kamon J, Minokoshi Y, Ito Y, Waki H, Uchida S, Yamashita S, Noda M, Kita S, Ueki K, Eto K, Akanuma Y, Froguel P, Foufelle F, Ferre P, Carling D, Kimura S, Nagai R, Kahn BB, Kadowaki T: Adiponectin stimulates glucose utilization and fatty-acid oxidation by activating AMP-activated protein kinase. Nature Medicine 2002, 8:1288-1295.

25. Bassi M, do Carmo JM, Hall JE, da Silva AA: Chronic effects of centrally administered adiponectin on appetite, metabolism and blood pressure regulation in normotensive and hypertensive rats. Peptides 2012, 37:1-5.

26. Hoyda TD, Samson WK, Ferguson AV: Adiponectin depolarizes parvocellular paraventricular nucleus neurons controlling neuroendocrine and autonomic function. Endocrinology 2009, 150:832-840.

27. Barbosa IG, Rocha NP, de Miranda AS, Magalhaes PV, Huguet RB, de Souza LP, Kapczinski F, Teixeira AL: Increased levels of adipokines in bipolar disorder. J Psychiatr Res 2012, 46:389-393.

28. Diniz BS, Teixeira AL, Campos AC, Miranda AS, Rocha NP, Talib LL, Gattaz WF, Forlenza OV: Reduced serum levels of adiponectin in elderly patients with major depression. J Psychiatr Res 2012, 46:1081-1085.

29. Liu J, Guo M, Zhang D, Cheng S-Y, Liu M, Ding J, Scherer PE, Liu F, and XinYun Lu: Adiponectin is critical in determining susceptibility to depressive behaviors and has antidepressant-like activity. Proc Natl Acad Sci USA 2012, 109:12248-12253.

30. Schwartz DR, Lazar MA: Human resistin: found in translation from mouse to man. Trends Endocrinol Metab 2011, 22:259-265.

31. Krysiak R: The role of adipokines in connective tissue diseases. Eur J Nutr 2012, 51:513-528.

32. Patel L, Buckels AC, Kinghorn IJ, Murdock PR, Holbrook JD, Plumpton C, Macphee $\mathrm{CH}$, Smith SA: Resistin is expressed in human macrophages and directly regulated by PPAR gamma activators. Biochem Biophys Res Commun 2003, 300:472-476.

33. Nagaev I, Bokarewa M, Tarkowski A, Smith U: Human resistin is a systemic immune-derived proinflammatory cytokine targeting both leukocytes and adipocytes. PLOS One 2006, 1:e31.

34. Kusminski CM, Scherer PE: The road from discovery to clinic: adiponectin as a biomarker of metabolic status. Nature 2009, 86:592-595

35. Lau CH, Muniandy S: Novel adiponectin-resistin (AR) and insulin resistance $\left(\mathrm{IR}_{\mathrm{AR}}\right)$ indexes are useful integrated diagnostic biomarkers for insulin resistance, type 2 diabetes and metabolic syndrome: a case control study. Cardiovascular Diabetology 2011, 10:8.

36. Tarkowski A, Bjersing J, Shestakov A, Bokarewa Ml: Resistin competes with lipopolysaccharide for binding to toll-like receptor 4. J Cell Mol Med 2010, 14:1419-1431.

37. Boström EA, Svensson M, Andersson S, Jonsson I-M, Ekwall A-K H, Eisler T, Dahlberg LE, Smith U, Bokarewa MI: Resistin and insulin/insulin-like growth factor signaling in rheumatoid arthritis. Arthritis Rheum 2011, 63:2894-2904.

38. Zhang Y, Proenca P, Maffei M, Barone M, Leopold L, Friedman JM: Positional cloning of the mouse obese gene and its human homologue. Nature 1994, 372:425-432.

39. Kamohara S, Burcelin R, Halaas JL, Friedman JM, Charron MJ: Acute stimulation of glucose metabolism in mice by leptin treatment. Nature 1997, 389:374-377.

40. Chehab FF: Leptin as a regulator of adipose mass and reproduction. Trends Pharmacol Sci 2000, 21:309-314.

41. Lu XY, Kim CS, Frazer A, Zhang W: Leptin: a potential novel antidepressant. Proc Natl Acad Sci USA 2006, 103:1593-1598.
42. Liu J, Garza JC, Bronner J, Kim CS, Zhang W, Lu XY: Acute administration of leptin produces anxiolytic-like effects: a comparison with fluoxetine. Psychopharmacology 2010, 207:535-545.

43. Satoh N, Ogawa Y, Katsuura G, Tsuji T, Masuzaki H, Hiraoka J, Okazaki T, Tamaki M, Hayase M, Yoshimasa Y, Nishi S, Hosoda K, Nakao K: Pathophysiological significance of the obese gene product, leptin, in ventromedial hypothalamus (VMH)-lesioned rats: evidence for loss of its satiety effect in VMH-lesioned rats. Endocrinology 1997, 138:947-954.

44. Delgado TC, Violante IR, Nieto-Charques L, Cerdan S: Neuroglial metabolic compartmentation underlying leptin deficiency in the obese ob/ob mice as detected by magnetic resonance imaging and spectroscopy methods. Journal of Cerebral Blood Flow \& Metabolism 2011, 31:2257-2266.

45. Gao S, Zhu G, Gao X, Wu D, Carrasco P, Casals N, Hegardt FG, Moran TH, Lopaschuk GD: Important roles of brain-specific carnitine palmitoyltransferase and ceramide metabolism in leptin hypothalamic control of feeding. Proc Natl Acad Sci USA 2011, 108:9691-9696.

46. Stephens TW, Basinski M, Bristow PK, Bue-Valleskey JM, Burgett SG, Craft L, Hale J, Hoffmann J, Hsiung HM, Kriauciunas A: The role of neuropeptide $Y$ in the antiobesity action of the obese gene product. Nature 1995 377:530-532.

47. Haque Z, Akbar N, Yasmeen F, Haleem MA, Haleem DJ: Inhibition of Immobilization Stress-induced Anorexia, Behavioral Deficits and Plasma Corticosterone Secretion by Injected Leptin in Rats. Stress 2012, doi:10.3109/10253890.2012.73604.

48. Guo M, Huang TY, Garza JC, Chua SC, Lu XY: Selective deletion of leptin receptors in adult hippocampus induces depression-related behaviours. Int I Neuropsychopharmacol 2012, 29:1-11.

49. Maeda T, Kiguchi N, Kobayashi Y, Ikuta T, Ozaki M, Kishioka S: Leptin derived from adipocytes in injured peripheral nerves facilitates development of neuropathic pain via macrophage stimulation. Proc Natl Acad Sci USA 2009, 106:13076-13081.

50. Malva JO, Xapelli S, Baptista S, Valero J, Agasse F, Ferreira R, Silva AP: Multifaces of neuropeptide $Y$ in the brain - neuroprotection, neurogenesis and neuroinflammation. Neuropeptides 2012, 46:299-308.

51. Anderberg UM, Liu Z, Berglund L, Nyberg F: Elevated plasma levels of neuropeptide $Y$ in female fibromyalgia patients. Eur J Pain 1999, 3:19-30.

52. Crofford LJ, Pillemer SR, Kalogeras KT, Cash JM, Michelson D, Kling MA, Sternberg EM, Gold PW, Chrousos GP, Wilder RL: Hypothalamic-pituitaryadrenal axis perturbations in patients with fibromyalgia. Arthritis Rheum 1994, 37:1583-1592.

53. Di Franco M, lannuccelli C, Alessandri C, Paradiso M, Riccieri V, Libri F, Valesini G: Autonomic dysfunction and neuropeptide $Y$ in fibromyalgia. Clin Exp Rheumatol 2009, 27:S75-78.

54. Griep EN, Boersma JW, Lentjes EG, Prins AP, van der Korst JK, de Kloet ER: Function of the hypothalamic-pituitary-adrenal axis in patients with fibromyalgia and low back pain. J Rheumatol 1998, 25:1374-1381.

55. Fletcher MA, Rosenthal M, Antoni M, Ironson G, Zeng XR, Barnes Z, Harvey JM, Hurwitz B, Levis S, Broderick G, Klimas NG: Plasma neuropeptide $\mathrm{Y}$ : a biomarker for symptom severity in chronic fatigue syndrome. Behav Brain Funct 2010, 6:76.

56. Klimas NG, Broderick G, Fletcher MA: Biomarkers for chronic fatigue. Brain, Behaviour, and Immunity 2012, 26:1202-1210.

57. Irwin MR: Human psychoneuroimmunology: 20 years of discovery. Brain behav 2008, 22:129-139.

58. Russel IJ, Orr MD, Litman B, Vipraio GA, Alboukrek D, Michalek JE, Lopez Y, MacKillip F: Elevated cerebrospinal fluid levels of substance $P$ in patients with the fibromyalgia syndrome. Arthritis Rheum 1994, 37:1593-1601.

59. Vaeroy $H$, Helle R, Forre O, Kass E, Terenius L: Elevated CSF levels of substance $P$ and high incidence of Raynaud phenomen in patients with fibromyalgi: new features for diagnosis. Pain 1988, 32:21-26.

60. Liu Z, Welin M, Bragee B, Nyberg F: A high-recovery extraction procedure for quantitative analysis of substance $P$ and opioid peptides in human cerebrospinal fluid. Peptides 2000, 21:853-860.

61. Giovengo SL, Russel IJ, Larson AA: Increased concentrations of nerve growth factor in cerebrospinal fluid of patients with fibromyalgia. $J$ Rheumatol 1999, 26:1564-1569.

62. Kadetoff D, Lampa J, Westman M, Andersson M, Kosek E: Evidence of central inflammation in fibromyalgia - increased cerebrospinal fluid interleukin-8 levels. J Neuroimmunol 2012, 242:33-38.

63. Mannerkorpi K, Nordeman L, Cider $\AA$, Jonsson G: Does moderate-to-high aerobic exercise result in better improvement of body impairments and 
pain than low-intensive exercise in FM? A prospective randomised controlled trial. Arthritis Res Ther 2010, 12:R189.

64. Wolfe F, Smythe HA, Yunus MB, Bennett RM, Bombardier C, Goldenberg DL, Tugwell P, Campbell SM, Abeles M, Clark P, Fam AG, Farber SJ, Fiechtner JJ, Franklin CM, Gatter RA, Hamaty D, Lessard J, Lichtbroun AS, Masi AT, McCain GA, Reynolds WJ, Romano TJ, Russell IJ, Sheon RP: The American College of Rheumatology 1990 criteria for the classification of fibromyalgia: report of the Multicenter Criteria Committee. Arthritis Rheum 1990, 33:160-172.

65. Burckhardt CS, Clark SR, Bennett RM: The fibromyalgia impact questionnaire: development and validation. J Rheumatol 1991, 18:728-733.

66. Ericsson A, Mannerkorpi K: Assessment of fatigue in patients with fibromyalgia and chronic widespread pain. Reliability and validity of the Swedish version of the MFI-20. Disabil Rehabil 2007, 30:1665-1670.

67. Srikuea R, Symons TB, Long DE, Lee JD, Shang Y, Chomentowski PJ, Yu G, Crofford $L$, Peterson CA: Fibromyalgia is associated with altered skeletal muscle characteristics which may contribute to post-exertional fatigue in post-menopausal women. Arthritis Rheum 2012, 65:519-528.

68. Carville S, Arendt-Nielsen S, Bliddal H, Blotman F, Branco JC, Buskila D, Da Silva JA, Danneskiold-Samsøe B, Dincer F, Henriksson C, Henriksson KG, Kosek E, Longley K, McCarthy GM, Perrot S, Puszczewicz M, Sarzi-Puttini P, Silman A, Späth M, Choy EH; EULAR: EULAR evidence-based recommendations for the management of fibromyalgia syndrome. Annals Rheum Dis 2008, 67:536-541.

69. Mannerkorpi K, Nordeman L, Ericsson A, Arndorw M, GAU-Study-Group: Pool-exercise for patients with fibromyalgia or chronic widespread pain. A randomized controlled trial and subgroup analyses. J Rehabil Med 2009, 41:751-760.

70. Kim CH, Luedtke CA, Vincent A, Thompson JM, Oh TH: Association of body mass index with symptom severity and quality of life in patients with fibromyalgia. Arthritis Care Res 2012, 64:222-228.

71. Mork PJ, Vasseljen O, Nilsen TI: Association between physical exercise, body mass index and risk of fibromyalgi: longitudinal data from the Norwegian Nord-Trondelag Health Study. Arthritis Care Res 2010, 62:611-617.

72. Choy E, Arnold L, Clauw D, Crofford L, Glass J, Simon L, Martin SA Strand CV, Williams DA, Mease PJ: Content and criterion validity of the preliminary core dataset for clinical trials in fibromyalgia syndrome. Rheumatol 2009, 36:2330-2334.

73. Bennett RM: Adult growth hormone deficiency in patients with fibromyalgia. Curr Rheumatol Rep 2002, 4:306-312.

74. Kohno D, Yada T: Neuropeptides. Arcuate NPY neurons sense and integrate peripheral metabolic signals to control feeding. Neuropeptides 2012, 46:315-319.

75. Garcia-Segura LM, Rodriguez JR, Torres-Aleman I: Localization of the insulilike growth factor I receptor in the cerebellum and hypothalamus of adult rats: an electron microscopic study. J Neurocytology 1997, 26:479-490.

76. Fernandez-Galas MC, Naftolin F, Garcia-Segura LM: Phasic synaptic remodeling of the rat arcuate nucleus during the estrous cycle depends on insulin-like growth factor-I receptor activation. J Neurosci Res 1999, 55:286-292.

77. Singhal NS, Lazar MA, Ahima RS: Central resistin induces insulin resistance via neuropeptide Y. J Neurosci 2007, 27:12924-12932.

doi:10.1186/ar4187

Cite this article as: Bjersing et al.: Exercise and obesity in fibromyalgia: beneficial roles of IGF-1 and resistin? Arthritis Research \& Therapy 201315 R34.

\section{Submit your next manuscript to BioMed Central and take full advantage of:}

- Convenient online submission

- Thorough peer review

- No space constraints or color figure charges

- Immediate publication on acceptance

- Inclusion in PubMed, CAS, Scopus and Google Scholar

- Research which is freely available for redistribution 